\title{
Pre-maximum spectro-imaging of the Mira star T Leporis with AMBER/VLTI ${ }^{\star}$
}

\author{
J.-B. Le Bouquin ${ }^{1}$, S. Lacour ${ }^{2}$, S. Renard ${ }^{2}$, E. Thiébaut ${ }^{3}$, A. Merand ${ }^{1}$, and T. Verhoelst ${ }^{4}$ \\ ${ }^{1}$ European Southern Observatory, Casilla 19001, Santiago 19, Chile \\ e-mail: jlebouqu@eso.org \\ 2 LAOG-UMR 5571, CNRS and Université Joseph Fourier, BP 53, 38041 Grenoble, France \\ 3 AIRI/Observatoire de Lyon, France and Jean-Marie Mariotti Center, France \\ ${ }^{4}$ Instituut voor Sterrenkunde, KULeuven, Celestijnenlaan 200D, 3001 Leuven, Belgium
}

Received 23 December 2008 / Accepted 4 February 2009

\section{ABSTRACT}

\begin{abstract}
Context. Diffuse envelopes around Mira variables are among the most important sources influencing the chemical evolution of galaxies. However they represent an observational challenge because of their complex spectral features and their rapid temporal variability. Aims. We aimed at constraining the exact brightness distribution of the Mira star T Lep with a model-independent analysis. Methods. We obtained single-epoch interferometric observations with a dataset continuous in the spectral domain $(\lambda=1.5-2.4 \mu \mathrm{m})$ and in the spatial domain (interferometric baselines ranging from 11 to $96 \mathrm{~m}$ ). We performed a model independent image reconstruction for each spectral bin using the MIRA software. We completed the analysis by modeling the data with a simple star+layer model inspired from the images.

Results. Reconstructed images confirm the general picture of a central star partially obscured by the surrounding molecular shell of changing opacity. At $1.7 \mu \mathrm{m}$, the shell becomes optically thin, with corresponding emission appearing as a ring circling the star. This is the first direct evidence of the spherical morphology of the molecular shell. Model fitting confirmed a spherical layer of constant size and changing opacity over the wavelengths. Rough modeling points to a continuum opacity within the shell, in addition to the $\mathrm{CO}$ and $\mathrm{H}_{2} \mathrm{O}$ features. Accordingly, it appeared impossible to model the data by a photosphere alone in any of the spectral bins.
\end{abstract}

Key words. techniques: interferometric - stars: AGB and post-AGB - stars: atmospheres - stars: individual: T Lep - stars: mass-loss

\section{Introduction}

Among the Asymptotic Giant Branch members, Mira stars are low-mass $\left(1 M_{\odot}\right)$, large-amplitude $(\Delta V \approx 9)$, long-period variables $(\approx 1 \mathrm{yr})$, evolving toward the planetary nebula and white dwarf phases. Their important mass-loss rate, of the order of $10^{-6} M_{\odot} /$ year, significantly affects their evolution and is one of the most important sources for the chemical enrichment of the interstellar medium. A better understanding of these late stages of stellar evolution may help shed light on this important player in the chemical evolution of galaxies. Due to their large diameters and high luminosities, Mira variables are a favorite target for observations at high angular resolution in the optical and nearinfrared (NIR) wavelengths. The wealth of aperture-masking and long baseline interferometric information has strongly advanced the study of molecule and dust formation (Perrin et al. 1999; Thompson et al. 2002; Woodruff et al. 2004; Ohnaka 2004; Ireland \& Scholz 2006; Ragland et al. 2006). The global picture is an onion-like structure with molecular layers and dust shells surrounding the photosphere, the latter animated by various activities (pulsations, convection, wave-shocks) crucial to explain the mass-loss process and the structural evolution.

To fully characterize the structure of a Mira star's atmosphere, one would need the complete intensity map at all wavelengths and all pulsation phases, recorded over a large number of pulsation cycles. The majority of recent studies concentrated on the spectral and/or the phase dependence. They overcame the

^ Based on observations collected at the VLTI, ESO-Paranal, Chile. issue of the exact brightness distribution by using either integrated quantities (such as diameter, Millan-Gabet et al. 2005; Woodruff et al. 2008), or the direct comparison of a few interferometric measurements with geometric (Mennesson et al. 2002; Perrin et al. 2004) or dynamic models of pulsating atmospheres (Ohnaka et al. 2006; Wittkowski et al. 2007, 2008). To study the brightness distribution, the difficulty lies in collecting a sufficient amount of spatial information in a period of time shorter than the typical life-time of the expected structures, and within spectral bins small enough to spectrally resolve the molecular bands (for which completely different intensity maps are expected).

In this work, we present a multi-wavelength, spatially resolved observation of the single star T Lep. This Mira variable has a spectral type of M6e-M9e and V-band magnitude varying from 7.3 to 14.3 (Samus et al. 2004). For the ephemeris, we use a Modified Julian Day of last maximum brightness $T_{0}=$ 54446 days and a pulsation period of 380 days (based on the last cycles observed by AAVSO), instead of the 368 days from Whitelock et al. (2000). The mass-loss has been estimated to be $7.3 \times 10^{-7} M_{\odot} /$ year by Loup et al. (1993). The most prominent spectral features expected in the NIR comes from water vapor and also $\mathrm{CO}$. The shapes and widths of these molecular bands depend on the stellar phase.

We present the dataset in Sect. 2, with a special emphasis on the observations and data reduction. Image reconstruction is presented and discussed in Sect. 3. We model the data in Sect. 4 with a simple star+layer model inspired by Perrin et al. (2004). 
Table 1. Observation log.

\begin{tabular}{cccc}
\hline \hline Date & T Lep phase & Baselines & Spectral range \\
\hline $2008-10-10$ & 0.80 & G2-G0-K0 & $1.5087-2.3191 \mu \mathrm{m}$ \\
$2008-11-04$ & 0.86 & D0-H0-G1 & $1.4287-2.4413 \mu \mathrm{m}$ \\
$2008-11-06$ & 0.87 & A0-D0-H0 & $1.4287-2.4238 \mu \mathrm{m}$ \\
$2008-11-10$ & 0.88 & E0-G0-H0 & $1.4287-2.4413 \mu \mathrm{m}$ \\
\hline
\end{tabular}

\section{Observations and data reduction}

Data were collected at the Very Large Telescope Interferometer (VLTI, Haguenauer et al. 2008) with the spectrograph AMBER (Petrov et al. 2007) covering simultaneously the J-, H- and K-bands with a spectral resolution of $R \approx 35$. Even though J-band fringes have been properly recorded in several observations, we decided to discard them from the analysis of this paper since the data quality is significantly worse than for longer wavelengths. The majority of the observations have been obtained by the FINITO fringe-tracker (Le Bouquin et al. 2008). When the conditions were unstable, we used FINITO in group-tracking mode (instead of phase-tracking). This mode reduces the sensitivity to unstable atmospheres at the cost of a clearly reduced instrumental contrast. We used 4 configurations of 3 Auxiliary Telescopes (ATs) each. Data for the A0-D0-H0, D0-H0-G1 and $\mathrm{E} 0-\mathrm{G} 0-\mathrm{H} 0$ configurations were obtained within a few days of each other while data with G2-G0-K0, extracted from the archive, was obtained about 1 month before as shown in Table 1.

Concerning the wavelength tables, we performed a rough absolute calibration by cross-correlating our observed spectra with an atmospheric model. We found a systematic offset of $0.08 \pm$ $0.02 \mu \mathrm{m}$ with respect to the initial AMBER table.

Observations of calibrator stars with smaller angular diameters were interleaved with observations of T Lep. We were able to reach an average observation frequency of about 25 min per calibrated point. This appeared to be crucial to properly sample the instrumental and atmospheric transfer function (TF). This also allowed us to manually discard the obvious outlier measurements without degrading the spatial coverage of our dataset too much. Additionally, we discarded all observations where the FINITO locking ratio was lower than $60 \%$. Raw visibility and closure phase values were computed using the latest public version of the amdlib package (version 2.2, Tatulli \& AMBER consortium 2007) and the yorick interface provided by the Jean-Marie Mariotti Center. We used our own software to calibrate the instrumental and atmospheric TF (see Fig. 1 for an example). We estimated the uncertainty on the TF with the dispersion between the consecutive points obtained for each observation of a calibration star. This dispersion generally dominated the other sources of error (mainly the diameter uncertainty of calibrators) and was propagated to the uncertainties on the calibrated visibilities and phases obtained for T Lep. Figure 2 shows the final UV-plane coverage of all observations that successfully passed all steps of the data reduction and calibration quality control. The east-west direction is favored because of the geometry of the telescope triplets used. Visibility curves are displayed in Fig. 3.

Looking at the interferometric data, we found that the general picture of $\mathrm{T}$ Lep significantly departs from a simple disk or Gaussian. It is composed of at least two different features: one has a characteristic size 5-7 mas and the other 11-16 mas. The exact size of these components and the flux ratio depend on the wavelength. This is qualitatively in agreement with a complex stellar atmosphere surrounded by molecular shells with wavelength-dependent opacity. Additionally, the non-zero

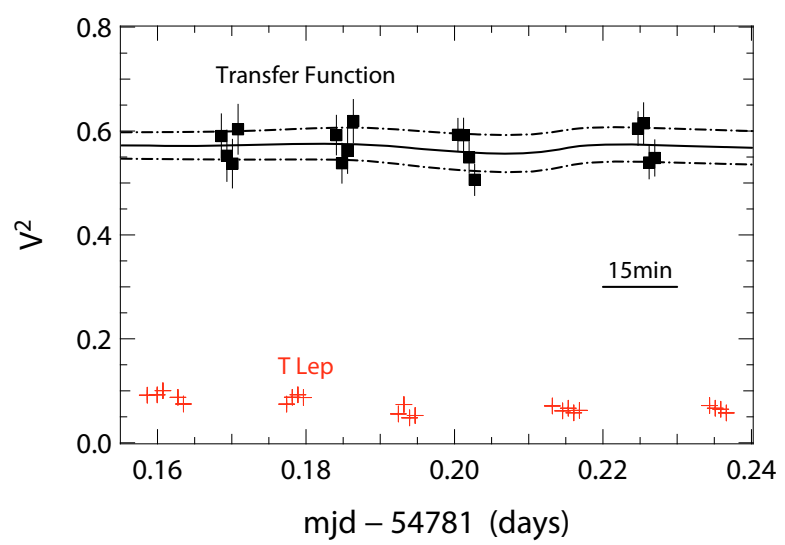

Fig. 1. Example of observation sequence, on the baseline G0-H0 and for the spectral bin $1.59 \mu \mathrm{m}$. The interpolated TF is represented.

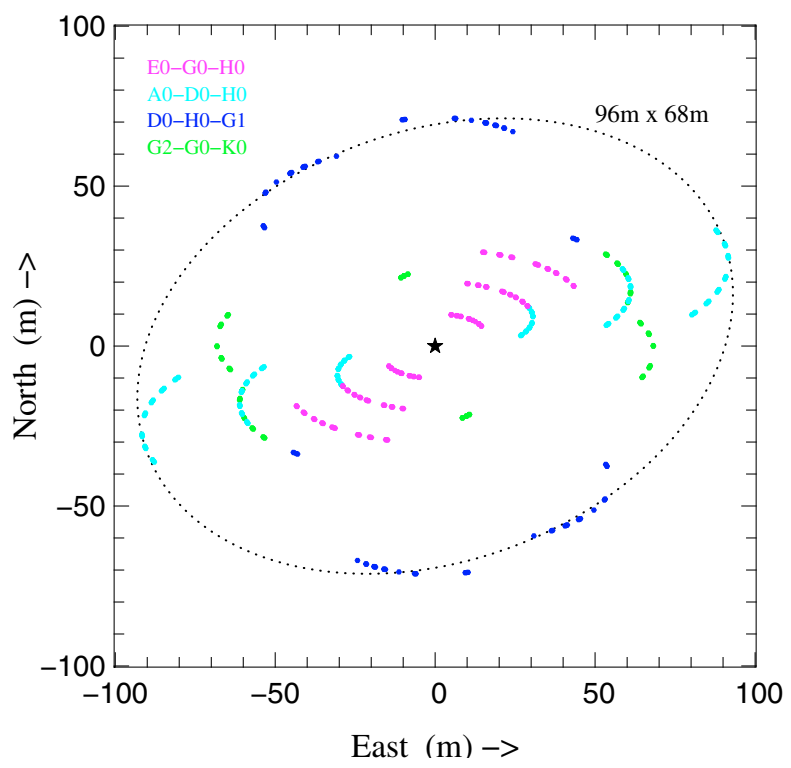

Fig. 2. Projected baselines (UV-plane) in meters of the complete AMBER dataset on T Lep.

closure phase (departure from $0-180 \mathrm{deg}$ up to $90 \mathrm{deg}$ in the $\mathrm{H}$-band and $30 \mathrm{deg}$ in the K-band) indicated the presence of asymmetries either in the shells or in the photosphere itself. Interestingly, we were not able to reproduce correctly the interferometric data with simple geometrical models such as Gaussian + Gaussian, or limb-darkened disk (LDD) + Gaussian, even when disregarding the closure phases. This was our main motivation to attempt to reconstruct an image.

\section{Image reconstruction}

Our dataset is perfectly suited to spectro-spatial inversion, where the spectral and the spatial dimensions are treated together assuming a certain number of degrees of freedom in each space. Unfortunately the necessary algorithms are not yet available, and we had to solve the problem independently for each spectral bin. We performed the image reconstruction with the MIRA software (Thiebaut 2008; Cotton et al. 2008). In spite of the apparent completeness of the dataset, we expected that it would not be straightforward to produce images: (i) the geometry of the VLTI array makes the east-west direction privileged; (ii) the necessary use of closure phases makes the phase information three times less rich than that of the visibilities. Therefore, a 


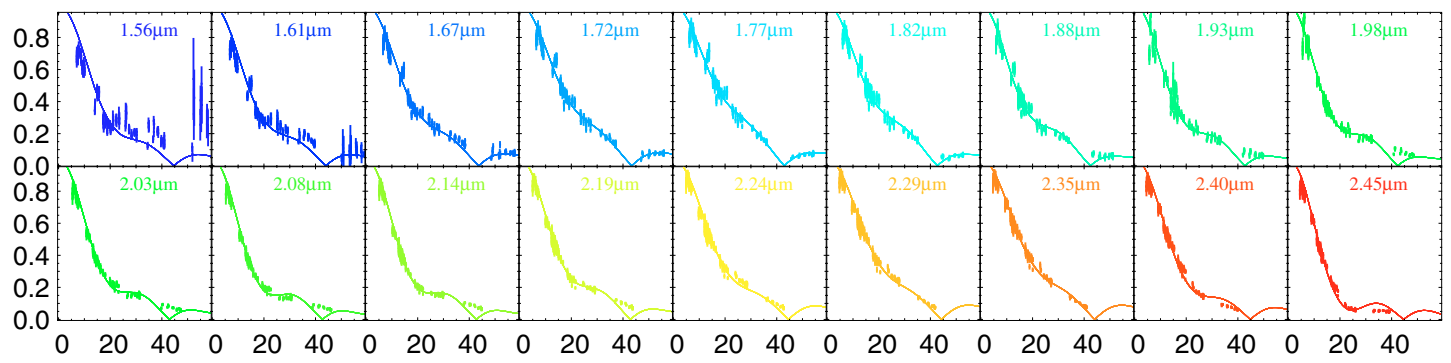

Fig. 3. Visibility profiles $V\left(B_{\lambda}\right)$ from AMBER/VLTI, compared to the star+layer model of Perrin et al. (2004). The horizontal axes are the spatial frequencies $B_{\lambda}$, marked in meter per micron, the vertical axes are the linear visibilities.
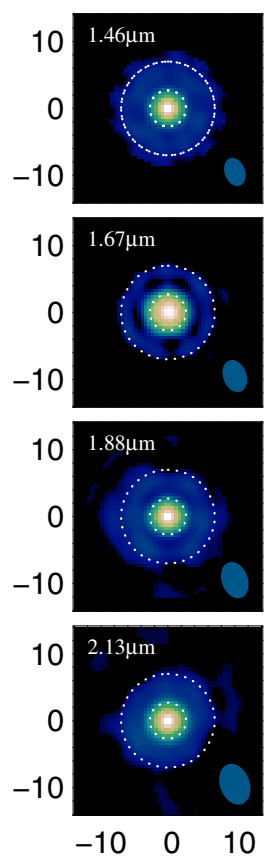
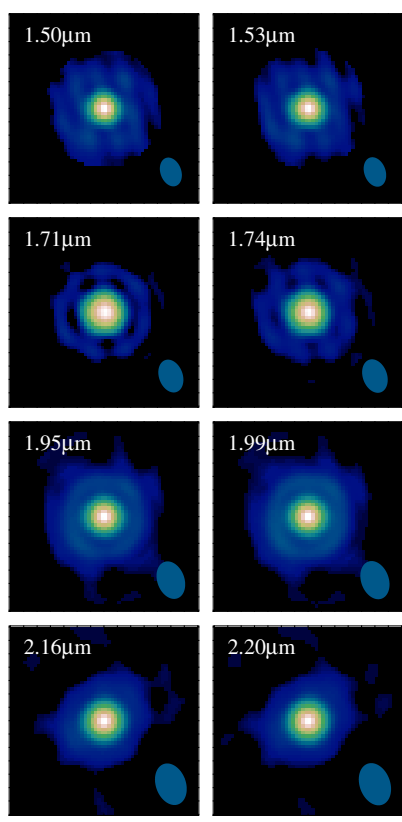
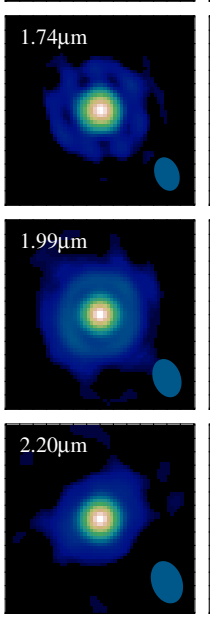
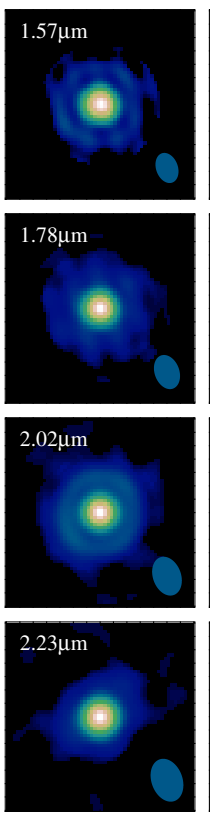
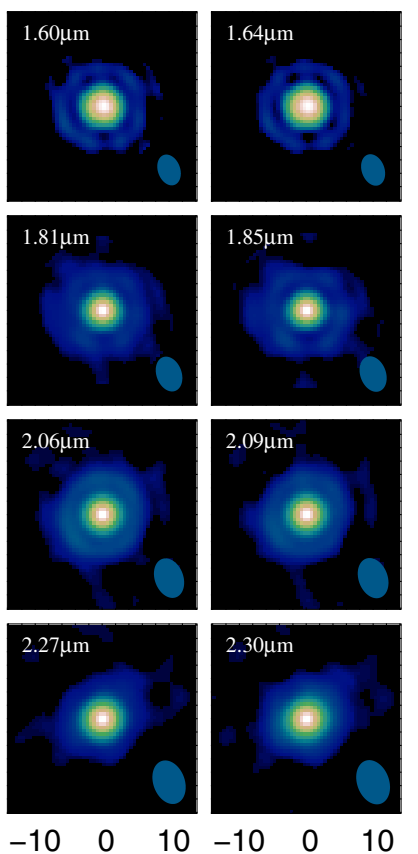

Fig. 4. Reconstructed images of T Lep with the MIRA software for several AMBER spectral bins across the H and K bands. The interferometric beam size is displayed in the bottom-right part of each image. Spatial scale is in mas. The white circles in the first column represent the average radius for the molecular layer $\left(\Phi_{l} \sim 15\right.$ mas) and for the central star $\left(\Phi_{l} \sim 5.8\right.$ mas), extracted from the modeling of Sect. 4 . It corresponds to the respective diameters of 2.5 and 1 AU (assuming $5.95 \pm 0.70$ mas parallax from van Leeuwen 2007). The mean surface brightness ratio between the photosphere and the molecular environment is around $10 \%$.

2-step image reconstruction strategy was specifically elaborated. The first step consisted of building a radially symmetrical image from the data. Only the baseline length was considered. The amplitude and sign of these pseudo data (which are all real due to the assumed symmetry) were derived from the measured power spectrum and sign of the closure phases respectively. We then used MIRA with a strong smoothness regularization to reconstruct the first stage images. The second step uses these brightness distributions as a support for a quadratic regularization of a 2D brightness map (Le Besnerais et al. 2008). Each of the spectral channels was processed separately. Resulting final images are presented in Fig. 4.

The recontructed images clearly highlight two components. The brighter central component corresponds to the photosphere, with a linear radius of $100 R_{\odot}$ (assuming a parallax of 5.95 mas from van Leeuwen 2007). The second component is the molecular layer (molsphere), characterized by the high spectral dependency of its apparent morphology. Within the molecular absorption bands $(1.5-1.6 \mu \mathrm{m}, 1.75-2.15 \mu \mathrm{m}, 2.3-2.5 \mu \mathrm{m})$, the shell is optically thick and appears, projected toward the observer, as a circular disk. Between these bands, absorption by molecules is not as strong, and the shell becomes optically thin. It appears as a projected ring encircling the photosphere $(1.6-1.75 \mu \mathrm{m})$. The lack of data points in the second lobe at $2.2 \mu \mathrm{m}$ (see Fig. 3) prevented the algorithm from reconstructing the inner gap between the molecular shell and the photosphere. Instead, it resulted in a decreased contrast between the molsphere and the photosphere.

Each spectral bin has been reduced and imaged independently. These images are in good agreement. It provides a strong indication that the MIRA algorithm is not inverting the statistical noise present in the data. Possible correlations may still originate from the calibration noise (atmospheric turbulence) and the use of common calibrator stars. However, these sources of errors are unlikely to give rise to such a coherent pattern as observed in Fig. 4. These images are a strong argument for a shell-like geometry of the molecules within the atmosphere.

\section{Intensity profile modeling}

In addition to reconstructed images of the molecular shells, we used a simple radiative transfer model developed by Perrin et al. (2004) to derive the physical parameters of the system. 


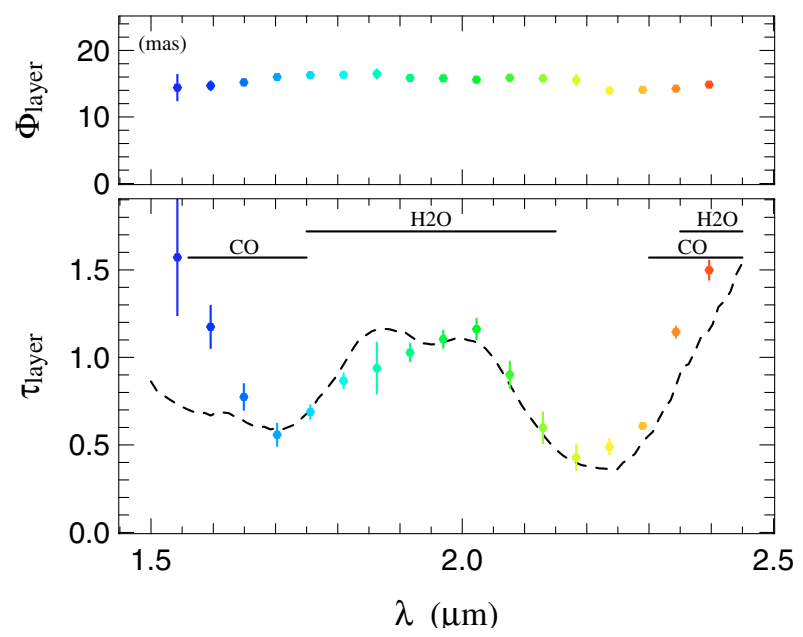

Fig. 5. Size and optical depth of the layer surrounding the photosphere, as extracted by a fit of the visibility curves (Sect. 4). The dashed curve is a fit with $\mathrm{H}_{2} \mathrm{O}$ and $\mathrm{CO}$ opacities assuming $T_{l}=1800 \mathrm{~K}$ (Verhoelst, private communication). A continuum opacity of $\tau \approx 0.2$ was added.

This model consists of a uniform disk star that emits radiation with a black body spectrum $\left(\Phi_{\star}, T_{\star}\right)$, surrounded by a spherical layer with no geometrical thickness. The layer is characterized by a size, a temperature and an optical depth $\left(\Phi_{l}, T_{l}, \tau_{l}\right)$. Such an analytic model can be compared to our wealth of observations without requiring time-consuming computations. We have also preferred to use an ad-hoc geometrical model rather than a self-consistent hydrodynamical model in order to easily give some geometrical flexibility to the fitting process. Other types of models will be discussed in further publications. We allowed the layer size and optical depth to be wavelength dependent, but not its temperature nor the stellar parameters. We were able to reproduce the visibility curves with the following parameters $T_{\star}=3500 \mathrm{~K}, T_{l}=1800 \mathrm{~K}, \Phi_{\star}=5.8$ mas and the chromatic quantities of Fig. 5. According to the good overlay of the visibility curves in Fig. 3, all spectral features observed in Fig. 5 are real. However, our fit shows important correlations between $T_{\star}, T_{l}$ and the average level of $\tau_{l}(\approx 1)$. Additional spectroscopic inputs may be needed to provide definite measurements of these quantities.

The model is able to reproduce the wavelength dependency of the visibility break around $B_{\lambda}=20$. Our data strongly suggest that the star+layer geometry is a good proxy for the real intensity profile and strengthens the result we had obtained with our imaging technique. It also gives a strong argument suggesting that the opacity effect explains most of the apparent size changes, without requiring important changes in the layer size (which was wavelength-independent in previous studies).

Following computations of Verhoelst et al. (2006), we modeled the spectral dependency of the optical depth as a sum of water vapor (mainly contributing in the range $1.75-2.15 \mu \mathrm{m}$ ), and CO (below $1.75 \mu \mathrm{m}$ and above $2.3 \mu \mathrm{m}$ ). However, we had to add a continuum opacity of constant value $\approx 0.2$ to be able to fairly reproduce the observed curve. Accordingly, the optical depth of the layer is always greater than 0.4 over the complete spectral range: it is impossible to see the photosphere alone. The presence of this continuum opacity, as well as the discrepencies in the fit, will require adapted modeling before being understood.

The simple star+layer model was able to reproduce almost all the visibility points with $V>15 \%$ but clearly failed at higher spatial frequency. An important feature in the visibility curves is the increase of contrast with baseline observed around $B_{\lambda}=45$ for the spectral bins around $1.8 \mu \mathrm{m}$. It could be tempting to identify it as a "visibility second lobe" following a "visibility null" (confirmed by the closure phases). This visibility lobe is apparently no longer observed around $1.9 \mu \mathrm{m}$ and above. The only way to continuously remove a visibility null is i) to fill it with unresolved flux; or ii) to displace it to infinite spatial frequencies by smoothing the corresponding edge; and iii) to add important asymmetries so that the visibility becomes a complex number around the null. The two first cases are not plausible in our case because the visibility around $B_{\lambda}=45$ never reaches 0 in the entire dataset. We conclude that the visibility profiles cannot be explained by an axisymmetric distribution continuous in wavelength. Undoubtedly, such an effect would have been missed with a less complete spectral and/or spatial coverage.

\section{Conclusions}

We believe this letter presents the first interferometric observations of a Mira star with a dataset almost continuous in the spectral and in the spatial domain. Obtaining it in a short amount of time has been made possible by the use of the AMBER spectrograph associated with the relocatable ATs of VLTI.

Individual spectral channel image reconstruction was achieved using the MIRA software. It provides confirmation of the existence of a spherical molecular shell at $\approx 1$ AU of the photosphere. Between 1.5-2.4 $\mu \mathrm{m}$ we did not find any spectral region where the brightness profile of $\mathrm{T}$ Lep can be explained by a single Gaussian or a single LDD. Conversely, when using a simple radiative transfer model we never found an optical depth less than 0.4 for the layer surrounding the photosphere. Rough modeling points to a continuum emission within the molecular shell, in addition to the $\mathrm{CO}$ and $\mathrm{H}_{2} \mathrm{O}$ features.

Acknowledgements. J.B.L.B. thanks the complete VLTI team. We acknowledge the use of the AAVSO International Database, of the Smithsonian/NASA Astrophysics Data System, and of the Centre de Donnees Astronomiques de Strasbourg. All graphics were drawn with the freeware yorick.

\section{References}

Cotton, W., Monnier, J., Baron, F., et al. 2008, in SPIE Conf., 7013 Haguenauer, P., Abuter, R., Alonso, J., et al. 2008, in SPIE Conf., 7013 Ireland, M. J., \& Scholz, M. 2006, MNRAS, 367, 1585

Le Besnerais, G., Lacour, S., Mugnier, L. M., et al. 2008, in IEEE Journal of Selected Topics in Signal Processing

Le Bouquin, J.-B., Abuter, R., Bauvir, B., et al. 2008, in SPIE Conf., 7013 Loup, C., Forveille, T., Omont, A., \& Paul, J. F. 1993, A\&AS, 99, 291 Mennesson, B., Perrin, G., Chagnon, G., et al. 2002, ApJ, 579, 446 Millan-Gabet, R., Pedretti, E., Monnier, J. D., et al. 2005, ApJ, 620, 961 Ohnaka, K. 2004, A\&A, 424, 1011

Ohnaka, K., Scholz, M., \& Wood, P. R. 2006, A\&A, 446, 1119

Perrin, G., Coudé du Foresto, V., Ridgway, S. T., et al. 1999, A\&A, 345, 221

Perrin, G., Ridgway, S. T., Mennesson, B., et al. 2004, A\&A, 426, 279

Petrov, R. G., Malbet, F., Weigelt, G., et al. 2007, A\&A, 464, 1

Ragland, S., Traub, W. A., Berger, J.-P., et al. 2006, ApJ, 652, 650

Samus, N., Durlevich, O. V., et al. 2004, VizieR Online Data Catalog, 2250, 0

Tatulli, E., \& AMBER consortium 2007, A\&A, 464, 29

Thiebaut, E. 2008, in SPIE Conf., 7013

Thompson, R., Creech-Eakman, M. J., \& van Belle, G. 2002, ApJ, 577, 447 van Leeuwen, F. 2007, Hipparcos, the new reduction of the raw data (Springer) Verhoelst, T., Decin, L., van Malderen, R., et al. 2006, A\&A, 447, 311 Whitelock, P., Marang, F., \& Feast, M. 2000, MNRAS, 319, 728

Wittkowski, M., Boboltz, D. A., Ohnaka, K., et al. 2007, A\&A, 470, 191 Wittkowski, M., Boboltz, D. A., Driebe, T., et al. 2008, A\&A, 479, L21 Woodruff, H. C., Eberhardt, M., Driebe, T., et al. 2004, A\&A, 421, 703 Woodruff, H. C., Tuthill, P. G., Monnier, J. D., et al. 2008, ApJ, 673, 418 